\title{
EDITORIAL
}

\section{A Tip of the Hat to Our Peer Reviewers}

\author{
Michael A. Ashby $•$ Leigh E. Rich
}

Published online: 11 October 2011

(C) Springer Science+Business Media B.V. 2011

Serving as a peer reviewer for one or more papers in an academic journal is often a time-consuming but anonymous (and thankless!) task. Regardless, peer-reviewed journal articles continue to be the "golden" standard in academia, enabling researchers not only to share findings with colleagues across both borders and disciplines but also to earn institutional credit toward tenure and promotion. Authors of articles do not deserve the entirety of the credit, however: peer reviewers play a crucial role in improving manuscripts in terms of content, accuracy, and significance.

M. A. Ashby $(\bowtie)$

Palliative Care and Persistent Pain Services,

Royal Hobart Hospital, Southern Tasmania Area Health

Service and School of Medicine, Faculty of Health

Sciences, University of Tasmania,

1st Floor, Peacock Building, Repatriation Centre,

90 Davey St,

Hobart, TAS 7000, Australia

e-mail: michael.ashby@dhhs.tas.gov.au

L. E. Rich

Department of Health Sciences (Public Health),

Armstrong Atlantic State University,

11935 Abercorn Street,

Savannah, GA 31419, USA

e-mail: leigh.rich@armstrong.edu
We at the Journal of Bioethical Inquiry - including all editors, members of our Editorial Board, and authors published in the $J B I$ - would like to instigate a new tradition of taking a moment to formally thank our peer reviewers. We will do so on a yearly basis by publishing a list of peer reviewer names in each volume's fourth issue. Although this list will appear at the end of a calendar year, due to the nature of the academic publication process (i.e., the several-month lag between submitting manuscripts to production and the printing of the actual journal), the list will include scholars who have peer-reviewed one or more articles from September of the previous year to September of the current year. The following list of reviewers, since it is our first, goes beyond this time-frame and includes those who have peer-reviewed for the $J B I$ approximately during the past five years (when the $J B I$ began using the online manuscript submission and peer-review system Editorial Manager).

To all of our kind reviewers, as Shakespeare wrote in Twelfth Night, we "can no other answer make but thanks, / And thanks" for your expertise, feedback, and time. We know "oft good turns / Are shuffled off with such uncurrent pay" (III.iii.15-17) but that, as G.B. Stern maintained, "[s]ilent gratitude isn't much use to anyone," either.

If Cicero was correct in that "there is no duty more obligatory than the repayment of kindness," then we as journal editors and journal authors are clearly in your debt. 
Judith Ajani

Stuart Allan

Radha Ameur

Ron Amundson

Pamela Andanda

Rachel Ankeny

Paul Appelbaum

Richard Ashcroft

Mark Aulisio

Leonard Bailey

Niels Becker

Zvi Beckerman

Belinda Bennett

Arthur Bradley

Andrew Bradstock

Katrina Bramstedt

Iain Brassington

Bruce Braun

Annette Braunack-Mayer

Samantha Brennan

Lisa Bridle

Laura Buccini

Lawrence Burns

Susan Campbell

Leslie Cannold

Terry Carney

Colleen Cartwright

Don Chalmers

Don Chalmers

Peter Chang

Nathan Cherney

Paul Cheung

Steve Clarke

Simon Cooper

Fiona Cram

Andrew Crowden

Richard Day

Immaculada de Melo-Martin

Martin Delatycki

Justin Denholm

Donna Dickenson

Deborah Diniz

Ros Diprose

Sue Dodds

Charles Douglas

Jocelyn Downie

Marlene Drysdale
Mary Edington

Marie Edwards

Martyn Evans

Hege Charlotte Faber

Thomas Faunce

Jennifer Flynn

Joel Frader

Gillian Fuller

Lance Gable

Sabina Gainotti

Lisa Geunther

Sandy Gifford

Lyn Gilbert

Michael Gill

Lynn Gillam

Grant Gillett

Errin Gilson

Sara Goering

Imogen Goold

Jill Gordon

Andrew Gunstone

Kim Hall

Anita Ho

Soren Holm

Claire Hooker

Karen Houle

David Hunter

Rob Irvine

Hussain Jamila

Jane Johnson

Richard Joyce

Beres Joyner

Betty Kafanelis

Isabel Karpin

Danny Kelly

Ian Kerridge

Eva Kittay

Paul Komesaroff

Jaro Kotalik

Emma Kowal

Alexander Lautensach

Terry Leahy

Morgan LeFay Holmes

Rose Leontini

Neil Levy

Wendy Lipworth

Miles Little 
Paul Longmore

Mianna Lotz

Michael Lowe

Tamra Lysaght

Adrian Mackenzie

Paul Ulhas Macneill

Roger Magnusson

Martin Maiden

Jeff Malpas

Francis Masiye

Chris Mayes

Pam McGrath

Janice McLaughlin

Carolyn McLeod

Ladelle McWhorter

Carolyn Michelle

Tina Miller

Kathleen Montgomery

Gavin Mooney

Barry Morris

Christian Munthe

Stuart Murray

Cat Myser

David Neil

Louise Newman

Ainsley Newson

Dianne Nicol

Garry Nieuwkamp

Johanna Oksala

Margaret Otlowski

Malcolm Parker

Michael Parker

Murray Parsons

Alan Petersen

Carlo Petrini

Neil Pickering

Leslie Pickering Francis

Jessica Pierce

Hans Pols

Joseph Pugliese

Eric Racine

Stuart Rennie

Bernadette Richards

Michael Robertson

Heidi Robertson

Bob Robinson

Wendy Rogers
Juliet Rogers

Debra Rolfe

Steven Rosenman

Philipa Rothfield

Christopher James Ryan

John Sadler

Peter Saul

Kristin Savell

Camilla Scanlan

Christopher Scanlon

Udo Schuklenk

Margaret Scrimgeour

Jackie Scully

Linnell Secomb

Michael Selgelid

Sue Sherwin

Susan Sherwin

Mary Simmerling

Anne Simmonds

Robert Sinnerbrink

Loane Skene

Roslyn Sorensen

Robert Sparrow

Merle Spriggs

Moira Stephens

Cameron Stewart

Susan Stryker

Nikki Sullivan

J.S. Swindell

Ann Taylor

Miriam Taylor Gomez

Samantha Thomas

Colin Thomson

Bernadette Tobin

Martin Tolich

Rosemarie Tong

Martin Van der Weyden

Anton Van Niekerk

Marcel Verweij

A.M. Viens

Cathy Waldby

Tom Walker

Garry Walter

David Wasserman

John Waugh

John Weckert

Lorna Weir 
Kandy White

Mary White

Kate White

Richard White

Peter Whitehouse

Dominic James Wilkinson

Derek Wilson
Elizabeth Wilson

John Wiltshire

Sarah Winch

Peter Woelert

Charles Wolfe

Joanna Zylinska 